\title{
Synthesis of coumarin sulfonamides and sulfonylurea
}

\author{
Martin Kováč, ${ }^{1}$ Andrea Sabatié, and Lubomír Floch \\ Department of Organic Chemistry, Faculty of Chemical Technology, Slovak University of \\ Technology, SK-812 37 Bratislava, Slovak Republic \\ E-mail:floch@chelin.chtf.stuba.sk
}

\section{Dedicated to Professor R.A. Abramovitch on the occassion of his $7^{\text {th }}$ birthday (received 08 May 01; accepted 15 Oct 01; published on the web 23 Oct 01)}

\begin{abstract}
4-Coumarinsulfonamide 5c and 4-hydroxy-3-coumarinsulfonamide $\mathbf{7 b}$, were prepared from 4hydroxycoumarin 1. Coumarinsulfonamide 5c was served as intermediate for the synthesis of $\mathrm{N}$ (isopropylphenyl)- $N$-(coumarin-4-sulfonyl)urea $\mathbf{9}$ and $N$-(4-bromphenyl-), $\mathbf{8 a}, \quad N$-(1,3,4thiadiazol-2-yl-), 8b, and $N$-(4-isopropylphenyl)-4-aminocoumarin 8c.
\end{abstract}

Keywords: Coumarin sulfonamides, coumarin sulfonylureas, aminocoumarins

\section{Introduction}

Sulfonylurea herbicides posses herbicidal activity at unprecendent levels combined with very low mammalian toxicity and desirable environmental properties. ${ }^{2}$ The synthesis and SAR-study of a great number of sulfonylureas have shown that maximum herbicidal activity is found in compound having an ortho substituted aryl group next to unmodified sulfonylurea bridge and with the heterocycle as a pyrimidine or 1,3,5-triazine with methyl or methoxy in the 4 and 6 positions. $^{3}$

\section{ARYL-SO 2 -NH-CO-NH-HETEROCYCLE}

Our research effort has been focused on substitution of the ARYL part of general formula $\mathbf{I}$ by the coumarin moiety.

A comprehensive review of the syntheses of sulfonylureas and their intermediates has been published by Beyer et. al. ${ }^{4}$ The overall synthesis of these sulfonylureas involves preparation of the sulfonamide and their coupling reactions with isocyanate derivatives. 


\section{Results and Discussion}

The coumarinsulfonamide $\mathbf{5 c}$ is a key intermediate that required to prepare the target product of type I. 4-Chlorocoumarin 2a was prepared from 4-hydroxycoumarin 1. It is known, that the selectivity of the reaction of $\mathbf{1}$ with $\mathrm{POCl}_{3}$ is low, ${ }^{6,7}$ because a considerable amount of 4-chloro$3,4^{\prime}, 3^{\prime}, 4^{\prime \prime}$-tercoumarin $\mathbf{3}$ was formed as a by-product. Our method improved the yield of the $\mathbf{2 a}$ and significantly decreased yield of the 3 . ${ }^{1} \mathrm{H}-\mathrm{NMR}$ of the $\mathbf{3}$ clearly shows three doublets with a typical ortho interaction constant $\left(5,5^{\prime}, 5^{\prime \prime}-\mathrm{H}\right)$ and a singlet for $3{ }^{\prime \prime}-\mathrm{H} .{ }^{13} \mathrm{C}-\mathrm{NMR}$ (DEPT) spectra indicate the presence of 14 quaternary carbons and 13 tertiary $(\mathrm{C}-\mathrm{H})$ ones. Quaternary carbons are in positions 3 and 4 on the two of three $\alpha$-pyrone skeletons. Mass spectrum showed a molecular weight of 468 , which was consistent with the elemental analysis.

The key intermediate, 4-isopropylthiocoumarin $2 \mathbf{c}$ was easily prepared from 4chlorocoumarin 2a using sodium 2-propanethiol. Oxidation of $\mathbf{2 c}$ by chlorine in acetic acid, and subsequent transformation of the sulfonylchloride $\mathbf{5 a}$ by different amines led to the sulfonamides $\mathbf{5 b}, \mathbf{5 c}$. Attempted oxidation of the 4-ethylthiocoumarin $\mathbf{2 b}$ under the same condition led mainly to the 3,4-dichlorocoumarin 4 .

The most selective and effective way to the sulfonamide $\mathbf{5 c}$ was via the preparation of N-tbutyl coumarinsulfonamide $\mathbf{5 b}$, with subsequent removal of the $t$-butyl group with trifluoroacetic acid (TFA).

Chlorosulfonation of $\mathbf{1}$ exclusively produced 3-coumarinsulfonic acid $\mathbf{6}$ which was further converted to sulfonamide $\mathbf{7 b}$ (Scheme 1).

A very convenient method for the preparation of sulfonylureas is the diazabicycloundecene (DBU) catalyzed condensation of an arylsulfonamide with a phenoxycarbamate. Other route (Meyer and Fory method) involved the heating of a phenylcarbamate of the sulfonamide with arylamines and heteroamines. ${ }^{5}$

According to the method reported by Meyer and Fory, we have obtained 4-substituted aminocoumarins 8a and $\mathbf{8 b}$ (Scheme 2).

The attempted condensation of coumarinsulfonamide 5c with 4-isopropylphenylisocyanate using diazabicyclononene (DBN) led only to 4-aminosubstituted coumarin 8c. The $\mathrm{SnCl}_{4}$ catalyzed reaction of $\mathbf{5 c}$ with the isocyanate provides desired sulfonylurea $\mathbf{9}$ (Scheme 3). 

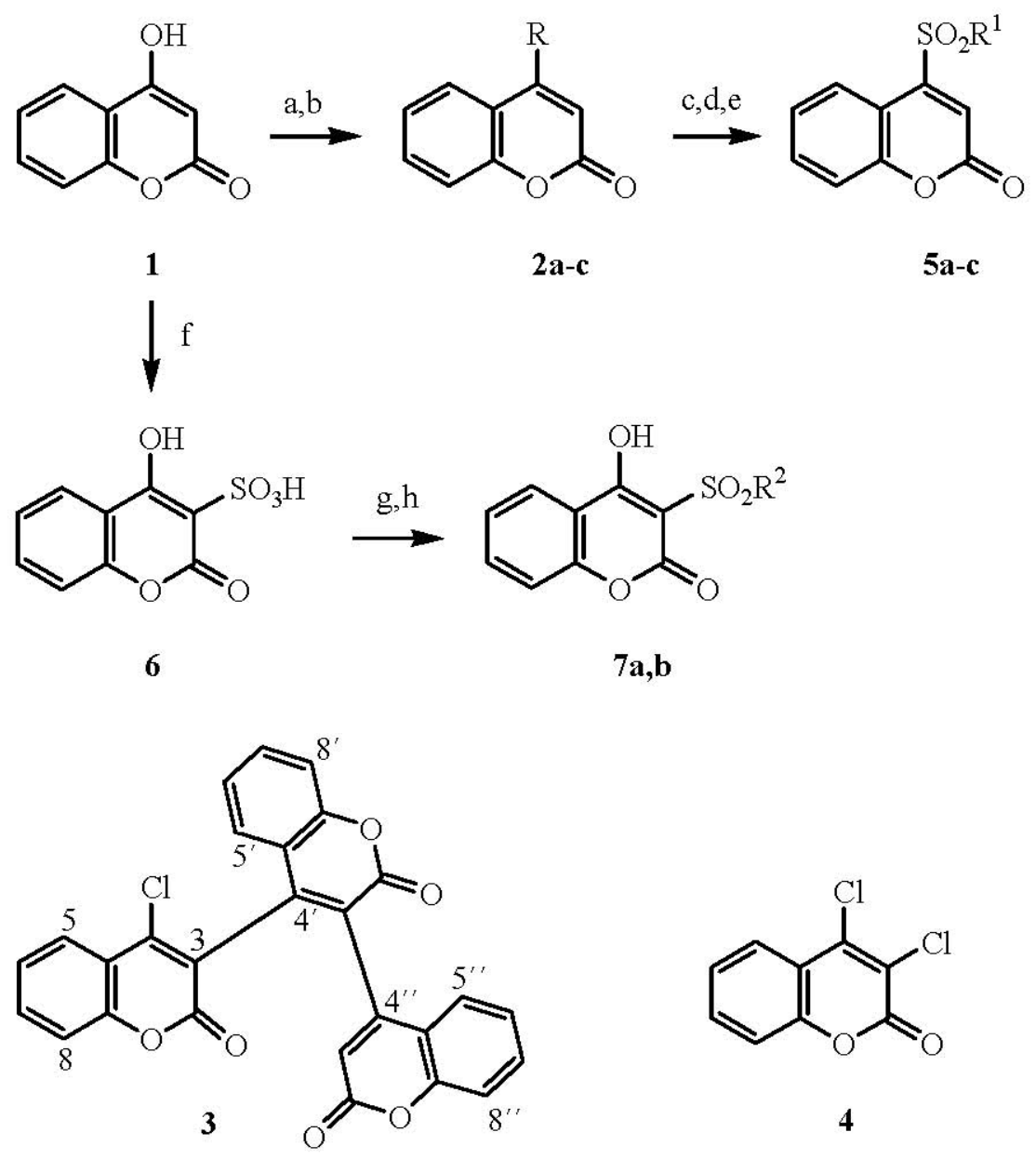

Scheme 1. (a) $\mathrm{POCl}_{3}, \mathrm{R}=\mathrm{Cl}$ 2a; (b) $\mathrm{RSNa} / \mathrm{MeOH}, \mathrm{R}=\mathrm{SC}_{2} \mathrm{H}_{5} \quad \mathbf{2 b}, \mathrm{i} / \mathrm{C}_{3} \mathrm{H}_{7} \mathrm{~S}$ 2 ; ; (c) $\mathrm{Cl}_{2} / \mathrm{AcOH} / \mathrm{H} 2 \mathrm{O}, \mathrm{R}^{1}=\mathrm{Cl} \mathbf{5 a}$; (d) $\mathrm{R}^{1}=\mathrm{t} / \mathrm{C}_{4} \mathrm{H}_{9} \mathrm{NH} \mathbf{5 b}$; (e) TFA, $\mathrm{R}^{1}=\mathrm{NH}_{2} \mathbf{5 c}$; (f) $\mathrm{ClSO}_{3} \mathrm{H}$; (g) $\mathrm{SOCl}_{2}, \mathrm{R}^{2}=\mathrm{Cl} 7 \mathbf{a} ;$ (h) $\mathrm{NH}_{3} / \mathrm{Et}_{2} \mathrm{O}, \mathrm{R}^{2}=\mathrm{NH}_{2} 7 \mathbf{b}$.

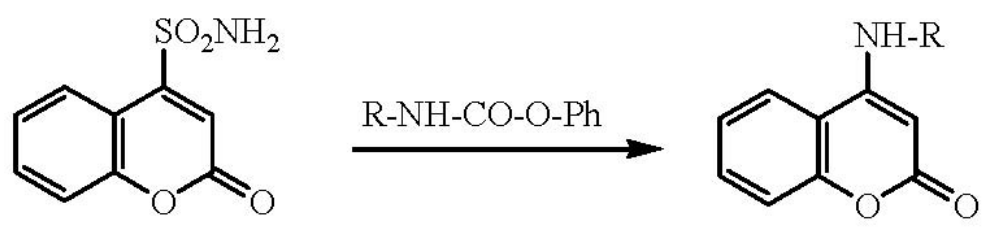

$5 c$

$\mathbf{8 a}, \mathbf{b}$

Scheme 2. R = 4-bromphenyl-, 8a; 1,3,4-thiadiazol-2-yl-, 8 b. 


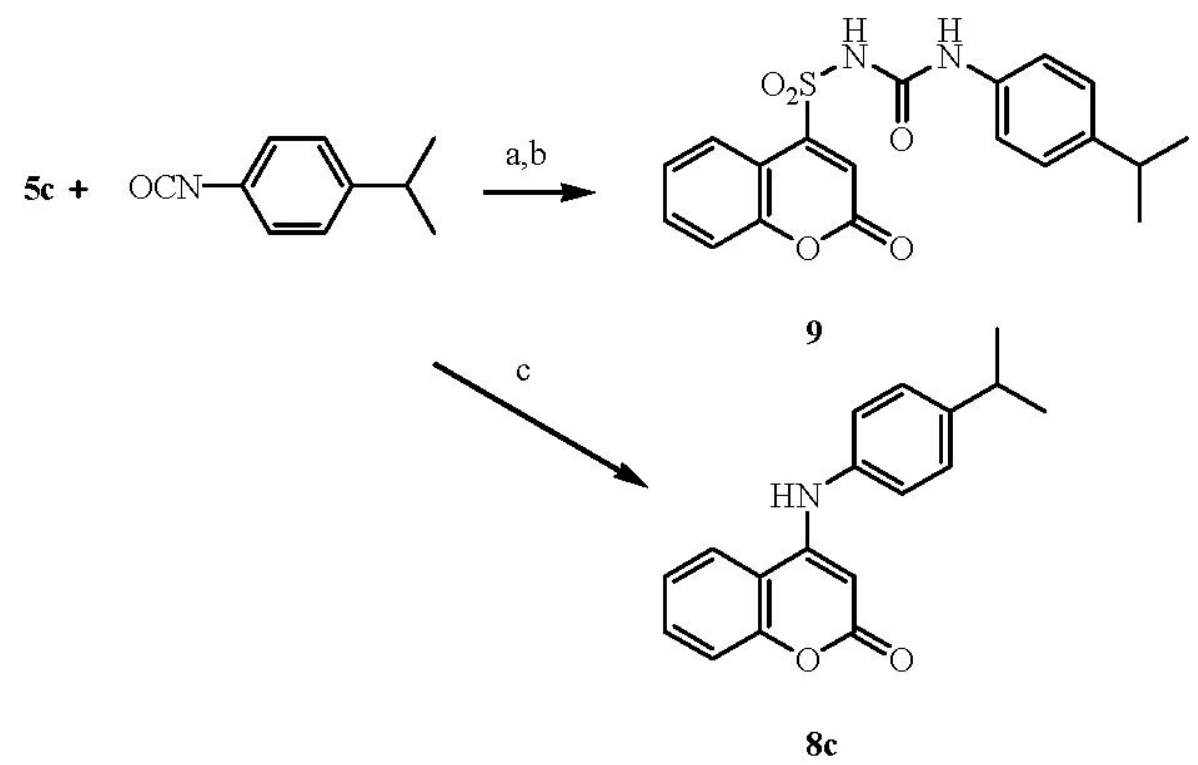

Scheme 3. (a) $\mathrm{SnCl}_{4}$; (b) $\mathrm{H}_{3} \mathrm{O}^{+}$; (c) $\mathrm{DBN}$

\section{Experimental Section}

General Procedures. Flash chromatography was carried out on $0.04-0.063 \mathrm{~mm}$ (Merck) silica gel, thin layer chromatography was carried out on aluminum back silica plates by Merck and plates were viewed in UV254 light. IR-spectra were recorded on a Philips Analytical PU 9800 spectrometer. ${ }^{1} \mathrm{H}-\mathrm{NMR}(300 \mathrm{MHz})$ and ${ }^{13} \mathrm{C}-\mathrm{NMR}(75 \mathrm{MHz})$ spectra were recorded on a Varian VXR 300 instrument at $293{ }^{\circ} \mathrm{K}$ in $\mathrm{CDCl}_{3}$ or DMSO D-6. Spectra were internally referenced to TMS. Peaks are reported in ppm downfield of TMS. Multiplicities are reported as singlet (s), doublet (d), triplet (t), quartet (q), some combinations of these were made by DEPT editing of the spectra. The MS-spectra were recorded on a AEI MS $902 \mathrm{~S}$ electron ionization spectrometer $(E I=70 \mathrm{eV})$. The elemental analysis, were performed on a Perkin-Elmer 2400 spectrometer.

Materials. The 4-hydroxycoumarin 1, ethanethiol, 2-propanethiol, trifluoroacetic acid (TFA), chlorosulfonic acid $\left(\mathrm{ClSO}_{3} \mathrm{H}\right), \mathrm{POCl}_{3}$ were purchased from Fluka, and $\mathrm{Bu}_{\mathrm{t}} \mathrm{NH}_{2}$ was purified, dried and distillated prior to use.

4-Chlorocoumarin (2a). 4-hydroxycoumarin 1 (30 g, $0.185 \mathrm{~mol})$ and $60 \mathrm{~mL} \mathrm{POCl}_{3}$ were refluxed for $1 \mathrm{~h}$, cooled, and slowly poured onto crushed ice (700 g) with vigorous stirring. The solid was collected by filtration and washed successively with ice-water. Azeotropic distillation with i-hexane, hot filtration of the by-product $(15 \mathrm{~g}, 17 \%){ }^{7}$ followed by evaporation of solvent and crystallization yielded $21.9 \mathrm{~g}(65 \%)$ of 4-chlorocoumarin with mp 87-89 ${ }^{\circ} \mathrm{C}$ (lit. $\left.{ }^{6} 89-91{ }^{\circ} \mathrm{C}\right)$. ${ }^{1} \mathrm{H}-\mathrm{NMR} \delta$ 7.88-7.30 (4H, m, ar-H), 6.62 (1H, s, 3-H); IR v 3098, 3069, 3040, 1754, 1721, 1611, 1603, 1449, 1348, 1273, $1177 \mathrm{~cm}^{-1}$; MS m/z: 182 (23), $180(\mathrm{MH}+, 77), 154$ (31), 152 (100), 89 (85), 63 (46), 62 (31), 39 (19). 
4-Chloro-3,4',3',4"'-tercoumarin (by-product) (3). Crystallization from acetic acid gave yellowish crystals, mp 321-325 ${ }^{\circ} \mathrm{C}$ (lit. $\left.{ }^{7} 324-327{ }^{\circ} \mathrm{C}\right) .{ }^{1} \mathrm{H}-\mathrm{NMR} \delta 8.11(1 \mathrm{H}, \mathrm{d}, J=7.92), 8.01$ $(1 \mathrm{H}, \mathrm{d}, J=7.92), 7.93(1 \mathrm{H}, \mathrm{d}, J=7.92), 7.81-7.41(9 \mathrm{H}, \mathrm{m}), 7.26\left(1 \mathrm{H}, \mathrm{s}, 3{ }^{\prime \prime}-\mathrm{H}\right) ;{ }^{13} \mathrm{C}-\mathrm{NMR} \delta$ $170.84,169.80,160.10,153.75,153.73,152.96,159.65,151.80,151.20,149.20,146.15,134.74$, $134.37,133.96,126.83,126.64,126.36,125.52,125.15,123.95,122.47,121.54,119.87,118.96$, 117.63, 117.21, 116.79; IR v 3088, 3068, 3039, 1718, 1625, 1593, 1538, 1352, $1187 \mathrm{~cm}^{-1}$; MS m/z: 470 (31), 468 (MH+, 86), 440 (9), 434 (18), 433 (56), 405 (22), 389 (13), 361 (11), 349 (8), 313 (36), 289 (100), 285 (36), 261 (11), 257 (13), 229 (16), 220 (7), 200 (20), 92 (9), 85 (29), 83 (40). Anal. Calcd for $\mathrm{C}_{27} \mathrm{H}_{13} \mathrm{ClO}_{6}$ : C, 69.16; H, 2.79. Found: C, 69.30; H, 2.75.

4-Ethylthiocoumarin (2b). To a stirred solution of 4-chlorocoumarin $(0.5 \mathrm{~g}, 2.8 \mathrm{mmol})$ in methanol $(10 \mathrm{~mL})$ was added dropwise sodium ethanethiol (prepared from Na $(0.07 \mathrm{~g}, 3 \mathrm{mmol})$ in $4 \mathrm{~mL}$ of methanol and $0.17 \mathrm{~g}(0.2 \mathrm{~mL}, 2.8 \mathrm{mmol})$ of ethanethiol). The mixture was refluxed for 30 minutes, followed by hot filtration. After cooling to $0{ }^{\circ} \mathrm{C}$ the product was filtered and dried $\left(60{ }^{\circ} \mathrm{C} / 15\right.$ torr). We have obtained $0.49 \mathrm{~g}(85 \%)$ of $\mathbf{2 b}$ with $\mathrm{mp} 111-114{ }^{\circ} \mathrm{C}$ (lit. ${ }^{8} 120-121$ $\left.{ }^{\circ} \mathrm{C}\right) .{ }^{1} \mathrm{H}-\mathrm{NMR} \delta 7.8-7.2(4 \mathrm{H}, \mathrm{m}, \mathrm{ar}-\mathrm{H}), 6.2(1 \mathrm{H}, \mathrm{s}, 3-\mathrm{H}), 3.1(2 \mathrm{H}, \mathrm{q}, J=7.5,11-\mathrm{H}), 1.5(3 \mathrm{H}, \mathrm{t}, J$ $=7.5,12-\mathrm{H})$; IR v 3067, 2967, 2909, 2868, 1761, 1730, 1607, 1447, 1350, 1260, $1165 \mathrm{~cm}^{-1}$.

4-Isopropylthiocoumarin (2c). Method A: To a refluxed solution of 4-chlorocoumarin (20 g, $0.11 \mathrm{~mol})$ in methanol $(350 \mathrm{~mL})$ was added dropwise sodium 2-propanethiol (prepared from 2.56 $\mathrm{g}$ of $\mathrm{Na}(0.11 \mathrm{~mol})$ in $150 \mathrm{~mL}$ of methanol and $8.44 \mathrm{~g}(10.3 \mathrm{~mL}, 0.11 \mathrm{~mol})$ of 2-propanethiol). The mixture was refluxed for 20 minutes, followed by hot filtration. After cooling to $0{ }^{\circ} \mathrm{C}$ the product was filtered and dried $\left(60^{\circ} \mathrm{C} / 15\right.$ torr). We have obtained $15.65 \mathrm{~g}(64 \%)$ of $2 \mathrm{c}$ with $\mathrm{mp}$ 123-125 ${ }^{\circ} \mathrm{C}$. Method B: A mixture of 4-chlorocoumarin $(1 \mathrm{~g}, 5.6 \mathrm{mmol}), \mathrm{Et}_{3} \mathrm{~N}(0.56 \mathrm{~g}, 0.8 \mathrm{~mL}$, $5.6 \mathrm{mmol})$, DMAP $(0.1 \mathrm{~g}, 0.8 \mathrm{mmol})$ and 2-propanethiol $(0.76 \mathrm{~g}, 0.9 \mathrm{~mL}, 10 \mathrm{mmol})$ in acetone $(10 \mathrm{~mL})$ was refluxed for $3.5 \mathrm{~h}$. After treating with $\mathrm{HCl}\left(4 \mathrm{~mL} \mathrm{HCl} / 30 \mathrm{~mL} \mathrm{H} \mathrm{H}_{2} \mathrm{O}\right)$, the formed solid was collected by filtration. Recrystallization from $\mathrm{MeOH}$ gave $0.34 \mathrm{~g}(28 \%)$ of product $2 \mathrm{c}$ with mp 121-124 ${ }^{\circ} \mathrm{C}$ (lit. $\left.{ }^{8} 131-132{ }^{\circ} \mathrm{C}\right) .{ }^{1} \mathrm{H}-\mathrm{NMR} \delta$ 7.7-7.2 (4H, m, ar-H), $6.2(1 \mathrm{H}, \mathrm{s}, 3-\mathrm{H}), 3.6$ (1H, k, 11-H), 1.5 (6H, d, 12-H, 12'-H); IR v 3061, 2975, 2926, 2826, 1755, 1705, 1601, 1595, 1545, 1346, 1192, $1180 \mathrm{~cm}^{-1}$; MS m/z: $220\left(\mathrm{MH}^{+}, 62\right), 221$ (10), 179 (10), 178 (100), 177 (28), 150 (52), 122 (14), 121 (22), 89 (14), 90 (14).

3,4-Dichlorocoumarin (4). Chlorine was added to a mixture of $\mathbf{2 b}(0.45 \mathrm{~g}, 2.2 \mathrm{mmol})$ and $1 \mathrm{~mL}$ of $\mathrm{H}_{2} \mathrm{O}$ in $4 \mathrm{~mL}$ of glacial acetic acid over 10 minutes at $10{ }^{\circ} \mathrm{C}$. After addition of $1 \mathrm{~mL}$ of $\mathrm{H}_{2} \mathrm{O}$ to the mixture, introducing of chlorine was continued for another 20 minutes. The formed solid was filtered and quenched with cold water. Recrystallization from $\mathrm{CHCl}_{3}$ gave $0.26 \mathrm{~g}(55 \%)$ of product with mp 107-108 ${ }^{\circ} \mathrm{C}$ (lit. $\left.{ }^{9} 106.7-107.5{ }^{\circ} \mathrm{C}\right)$. ; ${ }^{1} \mathrm{H}-\mathrm{NMR} \delta 7.88(1 \mathrm{H}, \mathrm{dd}, J=7.8,1.5,5$ $\mathrm{H}), 7.64(1 \mathrm{H}, \mathrm{td}, J=7.9,1.5,7-\mathrm{H}), 7.42(1 \mathrm{H}, \mathrm{td}, J=7.8,1.1,6-\mathrm{H}), 7.39(1 \mathrm{H}, \mathrm{d}, J=7.9,8-\mathrm{H})$; ${ }^{13} \mathrm{C}-\mathrm{NMR} \delta 155.89$ (2-C), 150.76 (9-C), 145.71 (4-C), 132.97 (7-C), 125.76 (5-C), 125.40 (6-C), 121.37 (3-C), 118.08 (10-C), 116.91 (8-C); IR v 3094, 3069, 3038, 1736, 1595, 1448, 1304, 1275, $1009 \mathrm{~cm}^{-1}$; MS m/z: 218 (34), 216 (68), $214\left(\mathrm{MH}^{+}, 100\right), 190$ (6), 188 (29), 186 (46), 160 (13), $158(20), 125$ (16), 123 (46). ${ }^{10}$

4-Coumarinsulfonyl chloride (5a). Chlorine (prepared in reaction between $\mathrm{K}_{2} \mathrm{Cr}_{2} \mathrm{O}_{7}(20 \mathrm{~g}$, 
$0.07 \mathrm{~mol})$ and $36 \% \mathrm{HCl}(80 \mathrm{~mL})$ was introduced into a mixture of $2 \mathrm{c}(6.5 \mathrm{~g}, 0.03 \mathrm{~mol})$ and $20 \mathrm{~mL}$ of $\mathrm{H}_{2} \mathrm{O}$ in glacial acetic acid $(50 \mathrm{~mL})$ over 20 minutes at $5-10{ }^{\circ} \mathrm{C}$. The formed solid was filtered and quenched with cold water. Recrystallization from $\mathrm{CHCl}_{3}$ provided $6.15 \mathrm{~g}(84 \%)$ of product with mp $119-121{ }^{\circ} \mathrm{C} ;{ }^{1} \mathrm{H}-\mathrm{NMR} \delta 8.29(1 \mathrm{H}, \mathrm{d}, J=7.9,5-\mathrm{H}), 7.73(1 \mathrm{H}, \mathrm{t}, J=7.3,7-\mathrm{H})$, 7.45 (2H, m, 6-H, 8-H), 7.21 (1H, s, 3-H); IR v 3073, 1759, 1732, 1607, 1450, 1385, 1366, 1198, $1167 \mathrm{~cm}^{-1}$; MS m/z: 246 (34), $244\left(\mathrm{MH}^{+}, 85\right), 218$ (11), 216 (26), 209 (64), 181 (11), 154 (13), 152 (40), 146 (21), 145 (66), 117 (15), 101 (100), 89 (74). Anal. Calcd for $\mathrm{C}_{9} \mathrm{H}_{5} \mathrm{ClO}_{4} \mathrm{~S}: \mathrm{C}, 44.18$; H, 2.06; S 13.13. Found: C, 44.32; H, 2.15; H, 12.96 .

$N$-(1,1-Dimethylethyl)-4-coumarinsulfonamide (5b). To a stirred and cooled solution at -10 ${ }^{\circ} \mathrm{C}$ of 4-coumarinsulfochloride $(2.46 \mathrm{~g}, 0.01 \mathrm{~mol})$ in $7 \mathrm{~mL}$ of $\mathrm{CHCl}_{3}$ was added dropwise the solution of $\mathrm{Bu}_{\mathrm{t}} \mathrm{NH}_{2}(1.46 \mathrm{~g}, 0.02 \mathrm{~mol})$ in $\mathrm{CHCl}_{3}(3.5 \mathrm{~mL})$ over 30 minutes. The reaction mixture was stirred 45 minutes at $20{ }^{\circ} \mathrm{C}$. Formed hydrochloride was separated by filtration. The solvent was removed and the product was crystallized from $\mathrm{MeOH}$. We have obtained $0.95 \mathrm{~g} \mathrm{(34 \% )} \mathrm{of}$ product with mp $135{ }^{\circ} \mathrm{C}$; ${ }^{1} \mathrm{H}-\mathrm{NMR} \delta 8.3-7.3(4 \mathrm{H}, \mathrm{m}, \mathrm{ar}-\mathrm{H}), 7.1(1 \mathrm{H}, \mathrm{s}, 3-\mathrm{H}), 4.9(1 \mathrm{H}, \mathrm{sbr}, \mathrm{NH})$, 1.4 (9H, s, 11-H); IR v 3231, 3090, 2982, 1759, 1717, 1607, 1341, 1196, 1154, $1007 \mathrm{~cm}^{-1}$; MS m/z: $281\left(\mathrm{MH}^{+}, 21\right), 266$ (100), 254 (4), 145 (12), 101 (27), 89 (12), 57 (30), 56 (65), 55 (25), 41 (99). Anal. Calcd for $\mathrm{C}_{13} \mathrm{H}_{15} \mathrm{NO}_{4} \mathrm{~S}$ : C, 55.50; H, 5.37; N, 4.98. Found: C, 55.40; H, 5.21; N, 4.90. 4-Coumarinsulfonamide (5c). A solution of $\mathrm{N}$-(1,1-dimethylethyl)-4-coumarinsulfonamide $(0.33 \mathrm{~g}, 1.2 \mathrm{mmol})$ in TFA $(10 \mathrm{~mL})$ was refluxed for $3 \mathrm{~h}$. The solvent was then rotary evaporated to leave a solid. Crystallization from $\mathrm{MeOH}$ gave $0.1 \mathrm{~g}(40 \%)$ of product 5c with $\mathrm{mp} 174-176$ ${ }^{\circ} \mathrm{C} ;{ }^{1} \mathrm{H}-\mathrm{NMR} \delta 8.24\left(2 \mathrm{H}, \mathrm{s}, \mathrm{NH}_{2}\right), 8.18(1 \mathrm{H}, \mathrm{d}, J=8.1,5-\mathrm{H}), 7.73(1 \mathrm{H}, \mathrm{t}, J=7.9,7-\mathrm{H}), 7.51(1 \mathrm{H}$, $\mathrm{d}, J=8.4,8-\mathrm{H}), 7.45(1 \mathrm{H}, \mathrm{t}, J=7.9,6-\mathrm{H}), 6.88(1 \mathrm{H}, \mathrm{s}, 3-\mathrm{H}) ;{ }^{13} \mathrm{C}-\mathrm{NMR} \delta 159.23$ (2-C), 153.7 (9C), 153.54 (4-C), 133.22 (7-C), 126.47 (5-C), 124.93 (6-C), 117.32, 115.44 (3-C, 8-C), 113.38 $(10-C)$; IR v 3304, 3220, 3086, 1763, 1720, 1603, 1448, 1344, 1203, $1163 \mathrm{~cm}^{-1}$; MS m/z: 225 (MH+, 89), 161 (5), 145 (24), 133 (21), 118 (100), 116 (13), 101 (36), 90 (18), 89 (47), 63 (34). Anal. Calcd for $\mathrm{C}_{9} \mathrm{H}_{7} \mathrm{NO}_{4} \mathrm{~S}: \mathrm{C}, 47.99 ; \mathrm{H}, 3.13 ; \mathrm{N}, 6.22 ; \mathrm{S}, 14.24$. Found: C, 48.19; H, 3.16; N, $6.47 ; \mathrm{S}, 14.08$.

4-Hydroxy-3-coumarinsulfonic acid (6). To a solution of $20 \mathrm{~mL}$ of $\mathrm{ClSO}_{3} \mathrm{H}$ in $80 \mathrm{~mL}$ of dioxane was added $1(24 \mathrm{~g}, 0.15 \mathrm{~mol})$ at $50{ }^{\circ} \mathrm{C} .{ }^{11}$ After 20 minutes the formed solid was filtered, washed with cold $\mathrm{Et}_{2} \mathrm{O}$ and air-dried. We have obtained $32.6 \mathrm{~g}(91 \%)$ of 6 with $\mathrm{mp} 92{ }^{\circ} \mathrm{C} ;{ }^{1} \mathrm{H}$ NMR $\delta 14.1\left(2 \mathrm{H}, \mathrm{s}_{\mathrm{br}}, \mathrm{OH}\right), 7.87(1 \mathrm{H}, \mathrm{d}, J=7.8,5-\mathrm{H}), 7.69(1 \mathrm{H}, \mathrm{t}, J=8.1,7-\mathrm{H}), 7.41-7.34(2 \mathrm{H}$, m, 6-H, 8-H), 2.56 (8H, s, dioxane- $\left.\mathrm{CH}_{2}\right) ;{ }^{13} \mathrm{C}-\mathrm{NMR} \delta 162.34$ (4-C), 157.03 (2-C), 152.57 (9-C), 133.61 (7-C), 124.44 (5-C), 124.19 (6-C), 116.27 (8-C), 114.9 (10-C), 107.71 (3-C), 66.38 (dioxane-C); IR $v$ 3500-2500, 1726, 1705, 1676, 1608, 1556, 1493, 1439, 1348, 1327, 1242, $1213,1157,1030 \mathrm{~cm}^{-1}$.

4-Hydroxy-3-coumarinsulfonamide (7b). Mixture of 6 (5 g, $21 \mathrm{mmol})$ and $\mathrm{SOCl}_{2}(20 \mathrm{~mL})$ was refluxed for $3 \mathrm{~h}^{11}$ After distillation of $\mathrm{SOCl}_{2}$, the residue was diluted with AcOEt. The sulfochloride $7 \mathbf{a}$ was filtered and air-dried (mp 125-145 $\left.{ }^{\circ} \mathrm{C}\right) .2 \mathrm{~g}$ of crude coumarinsulfochloride was added to a solution of $25 \% \mathrm{NH}_{3}$ in $\mathrm{MeOH}(5 \mathrm{~mL})$ at $-10{ }^{\circ} \mathrm{C}$. After 2 $\mathrm{h}$ of standing at room temperature, the volume of mixture was reduced for one-half and treated 
with $\mathrm{HCl}(5 \mathrm{~mL})$. The product was filtered and recrystallized from EtOH $(40 \mathrm{~mL})$. We have obtained $0.2 \mathrm{~g}(10 \%)$ of $7 \mathbf{b}$ with $\mathrm{mp} 238{ }^{\circ} \mathrm{C} ;{ }^{1} \mathrm{H}-\mathrm{NMR} \delta 7.96(1 \mathrm{H}, \mathrm{d}, J=7.4,5-\mathrm{H}), 7.76(1 \mathrm{H}, \mathrm{t}, J$ = 7.6, 7-H), 7.46-7.40 (2H, m, 6-H, 8-H), $4.50\left(3 \mathrm{H}, \mathrm{s}_{\mathrm{br}}, \mathrm{NH}_{2}, \mathrm{OH}\right) ;{ }^{13} \mathrm{C}-\mathrm{NMR} \delta 165.47$ (4-C), 157.16 (2-C), 153.0 (9-C), 135.03 (7-C), 125.05 (5-C), 124.84 (6-C), 116.76 (8-C), 115.24 (10C), 105.41 (3-C); IR v 3420, 3361, 3259, 3088, 1703, 1619, 1606, 1553, 1436, 1350, 1329, 1293 , $1126 \mathrm{~cm}^{-1}$; MS m/z: $241\left(\mathrm{MH}^{+}, 51\right), 224$ (37), 162 (11), 121 (39), 120 (100), 105 (7), 104 (5), 92 (34), 76 (10), 77 (10). Anal. Calcd for $\mathrm{C}_{9} \mathrm{H}_{7} \mathrm{NO}_{5} \mathrm{~S}: \mathrm{C}, 44.80 ; \mathrm{H}, 2.82$; N 5.83. Found: C, 44.98; $\mathrm{H}, 2.84 ; \mathrm{H}, 5.88$.

$N$-(4-Bromphenyl)-4-aminocoumarin $8 \mathrm{a}$ and $\mathrm{N}$-(1,3,4-thiadiazol-2-yl)-4-aminocoumarin (8b). A solution of 4-coumarinsulfonamide 5c $(0.1 \mathrm{~g}, 0.4 \mathrm{mmol})$, DBU $(0.06 \mathrm{~mL}, 0.4 \mathrm{mmol})$ and $0.4 \mathrm{mmol}$ of phenoxy $N$-(4-bromphenyl)carbamate or phenoxy $N$-(1,3,4-thiadiazol-2yl)carbamate was reflux for $3 \mathrm{~h}$. After evaporating of solvent (two third of volume), the residue was then stirred with the same volume of water contained $1 \mathrm{~mL}$ of $\mathrm{HCl}$. The formed solid products 8a or $\mathbf{8 b}$ was filtered and recrystallized from EtOH. We have obtained $59 \mathrm{mg}(42 \%)$ of 8a with mp $240{ }^{\circ} \mathrm{C}$ (subl.) or $89 \mathrm{mg}(82 \%)$ of $\mathbf{8 b}$ with mp $290{ }^{\circ} \mathrm{C}$; 8a: ${ }^{1} \mathrm{H}-\mathrm{NMR} \delta 9.33(1 \mathrm{H}, \mathrm{s}$, $\mathrm{NH}), 8.22(1 \mathrm{H}, \mathrm{d}, J=7.3,5-\mathrm{H}), 7.68-7.67(1 \mathrm{H}, \mathrm{m}, 7-\mathrm{H}), 7.66\left(2 \mathrm{H}, \mathrm{d}, J=8.6,12-\mathrm{H}, 12^{\prime}-\mathrm{H}\right)$, $7.42-7.40(2 \mathrm{H}, \mathrm{m}, 6-\mathrm{H}, 8-\mathrm{H}), 7.36\left(2 \mathrm{H}, \mathrm{d}, J=8.6,13-\mathrm{H}, 13^{\prime}-\mathrm{H}\right), 5.38(1 \mathrm{H}, \mathrm{s}, 3-\mathrm{H}) ;{ }^{13} \mathrm{C}-\mathrm{NMR} \delta$ 161.34 (2-C), 153.37 (9-C), 151.98 (4-C), 137.78 (11-C), 132.47 (7-C), 132.40 (13-C, 13'-C), 126.77 (12-C, $12^{\prime}$-C), 123.66 (5-C), 122.84 (6-C), 117.88 (14-C), 117.07 (8-C), 114.45 (10-C), 85.13 (3-C); IR v 3281, 3113, 3065, 1665, 1618, 1613, 1582, 1534, 1491, 1404, 1262, $1202 \mathrm{~cm}^{-1}$; MS m/z: 317 (100), 315 (MH+, 100), 300 (12), 298 (12), 289 (22), 287 (22), 275 (14), 273 (14), 260 (5), 258 (5), 247 (5), 245 (5), 236 (38), 235 (63), 208 (38), 180 (26), 157 (16), 155 (16), 118 (19), 96 (18), 90 (30). Anal. Calcd for $\mathrm{C}_{15} \mathrm{H}_{10} \mathrm{BrNO}_{2}$ : C, 56.98; H, 3.19; N, 4.32. Found: C, 55.84; H, 3.13; N, 4.32. 8b: ${ }^{1} \mathrm{H}-\mathrm{NMR} \delta 11.0(1 \mathrm{H}, \mathrm{s}$ r, $\mathrm{NH}), 9.23(1 \mathrm{H}, \mathrm{s}, 12-\mathrm{H}), 8.38(1 \mathrm{H}, \mathrm{d}, J=$ 7.32, 5-H), $7.69(1 \mathrm{H}, \mathrm{t}, J=7.5,7-\mathrm{H}), 7.5-7.4(2 \mathrm{H}, \mathrm{m}, 6-\mathrm{H}, 8-\mathrm{H}), 5.44(1 \mathrm{H}, \mathrm{s}, 3-\mathrm{H}) ;{ }^{13} \mathrm{C}-\mathrm{NMR} \delta$ 163.13 (4-C), 161.50 (2-C), 153.04 (9-C), 148.76 (11-C), 147.43 (12-C), 132.44 (7-C), 123.97 (6-C), 123.02 (5-C), 117.24 (8-C), 114.08 (10-C), 94.68 (3-C); IR v 3270, 3106, 3079, 3036, 1668, 1578, 1557, 1501, 1497, $1254 \mathrm{~cm}^{-1}$; MS m/z: $245\left(\mathrm{MH}^{+}, 41\right), 228$ (29), 217 (59), 190 (10), 175 (9), 163 (7), 144 (8), 132 (41), 127 (21), 124 (83), 123 (100), 103 (12), 101 (90), 96 (17), 89 (17), 74 (64), 69 (38), 68 (45). Anal. Calcd for $\mathrm{C}_{11} \mathrm{H}_{7} \mathrm{~N}_{3} \mathrm{O}_{2} \mathrm{~S}: \mathrm{C}, 53.86 ; \mathrm{H}, 2.88 ; \mathrm{N}, 17.13$. Found: C, 52.78; H, 2.81; N, 16.87 .

$\mathbf{N}$-(4-Isopropylphenyl)-4-aminocoumarin (8c). To a solution of 4-coumarinsulfonamide 5c $(0.5 \mathrm{~g}, 2.2 \mathrm{mmol})$ in $10 \mathrm{~mL}$ of dioxane was added 4-isopropylphenyl isocyanate $(0.53 \mathrm{~g}$, $3.3 \mathrm{mmol})$ and $\mathrm{DBN}(0.41 \mathrm{~g}, 0.4 \mathrm{ml}, 3.3 \mathrm{mmol})$ at $40{ }^{\circ} \mathrm{C}$, following the reflux for 15 minutes. After cooling, treating with $1 \mathrm{~mL}$ of $\mathrm{HCl}$ and removing of solvent, the product was purified by column chromatography $\left(\mathrm{SiO}_{2}, 40 \mathrm{~g} ; \mathrm{CHCl}_{3}\right)$. We have obtained $0.39 \mathrm{~g}(65 \%)$ of $\mathbf{8 c} ;{ }^{1} \mathrm{H}-\mathrm{NMR} \delta$ $9.28(1 \mathrm{H}, \mathrm{s}, \mathrm{NH}), 8.25(1 \mathrm{H}, \mathrm{d}, 5-\mathrm{H}), 7.64(1 \mathrm{H}, \mathrm{t}, 7-\mathrm{H}), 7.43-7.28(6 \mathrm{H}, \mathrm{m}, \mathrm{ar}-\mathrm{H}), 5.26$ (1H, s, 3$\mathrm{H}), 2.93(1 \mathrm{H}, \mathrm{k}, 15-\mathrm{H}), 1.23\left(6 \mathrm{H}, \mathrm{d}, 16-\mathrm{H}, 16^{\prime}-\mathrm{H}\right) ;{ }^{13} \mathrm{C}-\mathrm{NMR} \delta 161.47$ (2-C), 153.39 (9-C), 152.62 (4-C), 146.29 (11-C), 135.77 (14-C), 132.32 (7-C), 127.29 (13-C, 13'-C), 125.14 (12-C, 12'-C), 123.55 (6-C), 122.74 (5-C), 117.04 (8-C), 114.48 (10-C), 83.99 (3-C), 33.01 (15-C), 
24.02, 23.86 (16-C, 16'-C); IR v 3302, 3069, 3030, 2961, 2928, 1666, 1620, 1537, 1261, 1198 $\mathrm{cm}^{-1}$; MS m/z: $279\left(\mathrm{MH}^{+}, 86\right), 264$ (91), 237 (30), 236 (32), 146 (40), 145 (23), 135 (43), 128 (20), 120 (100), 91 (23), 77 (26). Anal. Calcd for $\mathrm{C}_{18} \mathrm{H}_{17} \mathrm{NO}_{2}$ : C, 77.42; H, 6.09; N, 5.02. Found: C, 76.82; H, 5.87; N, 4.98 .

$\mathrm{N}$-(4-isopropylphenyl)- $\mathrm{N}$-(coumarin-4-sulfonyl)urea (9). A mixture of 4-coumarinsulfonamide 5c $(0.5 \mathrm{~g}, 2.2 \mathrm{mmol})$, 4-isopropylphenyl isocyanate $(0.53 \mathrm{~g}, 3.3 \mathrm{mmol})$ and $\mathrm{SnCl}_{4}(0.4 \mathrm{~mL}$, $3.3 \mathrm{mmol})$ was heated to $(100 \pm 10){ }^{\circ} \mathrm{C}$ for $1.3 \mathrm{~h}$. After cooling the mixture was poured into ice-water $(10 \mathrm{~g})$ and $1 \mathrm{~mL}$ of $\mathrm{HCl}$ and $30 \mathrm{~mL}$ of AcOEt was added. The stirring was continued until two clear phases was disappeared. Organic layer was separated, dried and the solvent was evaporated. Purification by column chromatography $\left(\mathrm{SiO}_{2}, 50 \mathrm{~g} ; \mathrm{CHCl}_{3}\right)$ gave $0.39 \mathrm{~g}(46 \%)$ of 9 with mp 174-176 ${ }^{\circ} \mathrm{C} ;{ }^{1} \mathrm{H}-\mathrm{NMR} \delta 9.04(1 \mathrm{H}, \mathrm{s}, \mathrm{NH}), 8.26(1 \mathrm{H}, \mathrm{d}, 5-\mathrm{H}), 7.74(1 \mathrm{H}, \mathrm{t}, 7-\mathrm{H}), 7.55$ $(1 \mathrm{H}, \mathrm{d}, 8-\mathrm{H}), 7.50(1 \mathrm{H}, \mathrm{t}, 6-\mathrm{H}), 7.27\left(2 \mathrm{H}, \mathrm{d}, J=8.66,13-\mathrm{H}, 13^{\prime}-\mathrm{H}\right), 7.11(2 \mathrm{H}, \mathrm{d}, J=8.66,14-\mathrm{H}$, $\left.14^{\prime}-\mathrm{H}\right), 7.06(1 \mathrm{H}, \mathrm{s}, 3-\mathrm{H}), 4.02(1 \mathrm{H}, \mathrm{sbr}, \mathrm{NH}), 2.80(1 \mathrm{H}, \mathrm{k}, 16-\mathrm{H}), 1.12\left(6 \mathrm{H}, \mathrm{d}, 17-\mathrm{H}, 17^{\prime}-\mathrm{H}\right) ;{ }^{13} \mathrm{C}-$ NMR $\delta 158.55$ (2-C), 153.59 (9-C), 150.26 (4-C), 149.25 (11-C), 143.73 (12-C), 135.50 (15-C), $133.27(\mathrm{CH}), 126.54(2 \times \mathrm{CH}), 125.65(\mathrm{CH}), 124.97(\mathrm{CH}), 119.56(2 \times \mathrm{CH}), 118.33(\mathrm{CH})$, $117.42(\mathrm{CH}), 112.95$ (10-C), 32.81 (16-C), 24.05, 23.91 (17-C, 17'-C); IR v 3312, 3193, 3112, 2963, 2928, 1757, 1728, 1665, 1607, 1530, 1451, 1366, 1352, $1159 \mathrm{~cm}^{-1}$; MS m/z: 251 (6), 225 (3), 185 (3), 170 (9), 161 (6), 159 (8), 146 (9), 135 (31), 120 (100). Anal. Calcd for $\mathrm{C}_{19} \mathrm{H}_{18} \mathrm{~N}_{2} \mathrm{O}_{5} \mathrm{~S}: \mathrm{C}, 59.07$; H, 4.66; N, 7.25; S, 8.29. Found: C, 57.92; H, 4.64; N, 7.18; S, 8.37.

\section{Acknowledgements}

The authors wish to thank the Novartis Crop Protection AG, Basle (Switzerland), for their financial support of this research.

We also thank the Slovak Grant Agency, Slovak republic for financial support of this work (Grant No: 1/8109/01).

\section{References and Notes}

1. Part of the Master Thesis of M. K., Slovak University of Technology, Bratislava 1998.

2. Levitt, G. In Synthesis and Chemistry of Agrochemicals II; Baker, D. R.; Fenyes, J. G.; Moberg, W. K. Eds; ACS Symposium Series, 1991, 443, p 16.

3. Hay, J. V. Pestic. Sci. 1990, 29, 247.

4. Beyer, E. M., Jr.; Duffy, M. J.; Hay, J. V.; Schleuter, D. D. "Sulfonylureas" In Herbicides. Chemistry, Degradation and Mode of Action; Kearney, P. C.; Laufman, D. D. Eds; Marcel Dekker Inc., 1988.

5. Meyer, W.; Fory, W. U. S. Pat. 1983, 4 419, 121; Chem. Abstr. 1984, 100, 139141y.

6. Spalding, D. P.; Mosher, H. S.; Whitmore, F. C. J. Am. Chem. Soc. 1950, 72, 5338. 
7. Knight, A. R.; McIntyre, J. S. Can. J. Chem. 1968, 46, 2495.

8. Peinhardt, G.; Reppel, L. Pharmazie 1970, 25, 68.

9. Newman, M. S.; Schiff, S. J. Am. Chem. Soc. 1959, 81, 2266.

10. Zacharov, P. I. Zhurnal Org. Khimii 1971, 7 (2), 388.

11. Checchi, S.; Vettori, L. P.; Alberti, M. B. Gazz. Chim. Ital. 1967, 97, 1749. 